\title{
Obstacle Detection and Mapping System
}

\author{
Tsai-Hong Hong, Steven Legowik, and Marilyn Nashman \\ Intelligent Systems Division, National Institute of Standards and Technology (NIST)
}

Bldg. 220, Rm. B124, Gaithersburg, MD 20899 


\section{Introduction}

The ability to detect and avoid obstacles is a prerequisite for the success of the Demo III Unmanned Ground Vehicles (UGV) program. The near term goals of the project are to be able to drive the High Mobility Multipurpose Wheeled Vehicle (HMMWV) autonomously on cross-country roads with the ability to:

1. Drive autonomously at speeds up to 10 m.p.h., controlled as appropriate for the terrain and vehicle dynamics.

2. Detect obstacles and rough terrain conditions in time to enable deceleration to a safe speed or the ability to steer around the problem condition.

3. Maintain a control station with an interface for limited mission planning and data collection.

This paper discusses an obstacle detection algorithm developed at NIST in support of the obstacle detection and rough terrain conditions. The algorithm is a hybrid of grid-based and sensor-based obstacle detection and mapping techniques. The perception and obstacle detection/mapping module is part of the integrated 4D-Realtime Control System (RCS) system [1][2]. It consists of two sections: an obstacle detection section and a mapping section. The obstacle detection section processes range data read from a Ladar sensor. The algorithm converts range data into Cartesian coordinates in the sensor coordinate frame, and uses this information to detect obstacles. The second section, the mapping module, projects obstacle points onto a grid-based map. The map is used by the 4D-RCS planner module [11] to generate a traversable path for the vehicle. We have demonstrated autonomous driving with obstacle detection and avoidance on the NIST grounds and the Nike site at speeds of up to $24 \mathrm{~km} / \mathrm{h}$. 
Section 2 describes the sensors used in the 4D-RCS autonomous driving system. Section 3 describes in more detail the Ladar sensor's characteristics. Section 4 describes the obstacle detection algorithm. Section 5 evaluates the algorithm performance on both artificial and natural obstacles. Section 6 briefly describes the obstacle mapping module. In Section 7, we present our conclusions and plans for future work.

\section{System Sensors}

The sensors used in the system include a Dornier 1.2 Hz. Ladar Range Imaging Camera (EBK); the Ashtech Z12, a Global Positioning System (GPS) sensor, and an Inertial Navigation System (INS) sensor [14]. The inertial sensor is the U.S. Army's Modular Azimuth and Positioning System (MAPS) which contains three ring laser gyros, three accelerometers, and a rear axle odometer. All sensors are mounted on the HMMWV shown in Figure 1. The vehicle is equipped with electric actuators added to the steering, brake, transmission, transfer case, and parking brake. Dashboard-type feedback provides the controller with RPM, speed, temperature, fuel level, etc. Multiple navigation sensors are used. A Kalman filter [9] computes vehicle position and orientation using data from an inertial dead reckoning system and a carrier phase differential GPS unit, ${ }^{1}$

1. Certain commercial equipment, instruments, or materials are identified in this paper in order to adequately specify the experimental procedure. Such identification does not imply recommendation or endorsement by NIST, nor does it imply that the materials or equipment identified are necessarily best for the purpose. 


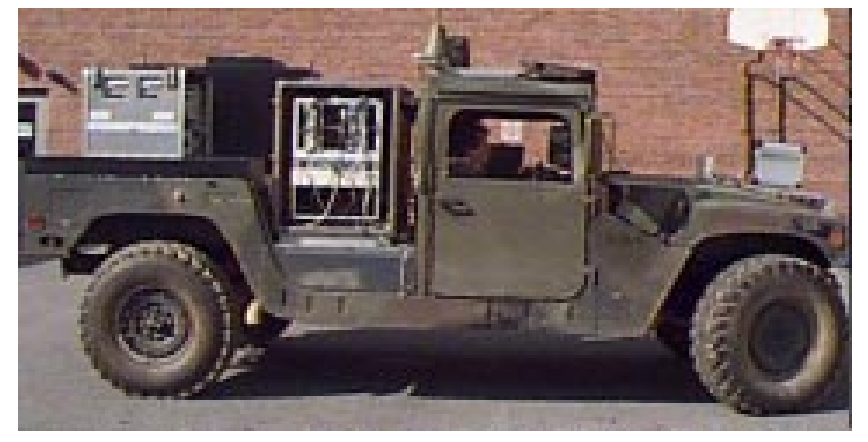

Figure 1. NIST HMMWV

\section{The EBK Ladar sensor}

We are using the EBK Ladar sensor as the primary obstacle detection sensor for autonomous driving. Table 1 shows a summary of the characteristic parameters of Dornier's $1.2 \mathrm{~Hz}$ Ladar camera. The vertical pixel spacing is mechanically fixed at 0.5 degrees by the spacing of the fiber optic array. This gives a vertical field of view of 31.5 degrees. The exact horizontal field of view and pixel spacing may vary slightly because the horizontal and vertical scans are not mechanically linked. However, they can be calculated exactly from scan mirror position data encoded in the image. The nominal value for the horizontal field of view is 60 degrees. In addition, there may be localized variations of the horizontal angular position of the image pixels due to mechanical disturbances of the sensor, but these can be detected and accounted for in range computations. The range image is generated by scanning a laser beam over the field of view. At each point in the scan, a pulse of laser light is emitted. This pulse is reflected back to the sensor from the object in its line of flight. The range of the object can be calculated from the light pulse's time of flight. Because the measurement uses time of flight rather than phase detection, the sensor produces an absolute value with no range ambiguity. Due to the short duration of the laser pulse, the EBK is eye-safe. 
The laser is scanned horizontally across the field of view by an oscillating mirror. The beam is scanned vertically by a nutating mirror which reflects the beam through a circular fiber optic array. This fiber optic array converts the circular motion of the nutating mirror into a linear scan in the image plane. Additional technical information about the EBK can be found in [3][4].

\subsection{Effects of Different Materials on Range Data}

We have examined the sensor's ability to measure different materials in order to determine its sensitivity. In order for the sensor to receive a laser pulse reflection, the reflection must be diffuse, i.e., the reflected laser light must travel in all directions from the target, one of which is back to the sensor. A highly specular surface may produce a false range reading.

\subsubsection{Absorptive Materials}

A false detection can occur when the surface of an object absorbs so much of the incident light that the returned light pulse does not have enough energy to trigger the receiver. Examples of absorptive materials are black clothing, dark hair, painted black stripes, the black baseboard along a wall, and very dark tar stains on asphalt. The sensor does not receive a reflected signal from these objects within the time of flight window, which is set for a $50 \mathrm{~m}$ maximum range. When no reflected light pulse is detected, the invalid timing results in an invalid range value. Some black colored objects, however, do show up. Automobile tires and black pavement return sufficient light to trigger the detector. An object's visibility depends on its reflective properties at $910 \mathrm{~nm}$. In practice, most surfaces in natural and artificial scenes offer a return signal of sufficient intensity to calculate a range value. 
Table 1 EBK operational parameters

\begin{tabular}{|c|c|}
\hline EBK Characteristic Parameters & \\
\hline Laser Wavelength & $910 \mathrm{~nm}$ \\
\hline Optical peak power & $10 \mathrm{~W}$ \\
\hline Imaging Range & $5 \mathrm{~m}$ to $50 \mathrm{~m}$ (typical) \\
\hline Range Resolution & $0.06 \mathrm{~m}$ \\
\hline Frame Rate & $1 \mathrm{~Hz}$ \\
\hline Field of View & 31 degrees by 60 degrees \\
\hline Beam Divergence & 0.2 degrees \\
\hline Beam Spacing & 0.5 degrees \\
\hline Eye safe & class 1 , IEC 825 and DIN VDE 0837 \\
\hline \multirow[t]{2}{*}{ Scanning } & Vertical: fiber optics \\
\hline & Horizontal: oscillating mirror \\
\hline Operating Voltage & $24 \mathrm{VDC}(18 \mathrm{~V}-28 \mathrm{~V})$ \\
\hline Power Consumption & $110 \mathrm{~W}$ \\
\hline Data Interface & RS422 serial, $10 \mathrm{MBit} / \mathrm{sec}$ \\
\hline Weight & $15 \mathrm{~kg}$ \\
\hline Dimensions & $30 \mathrm{~cm} \times 28 \mathrm{~cm} \times 27 \mathrm{~cm}$ \\
\hline Operating Environment & IP65, $-10^{0} \mathrm{C}$ to $50^{0} \mathrm{C}$ \\
\hline
\end{tabular}

\subsubsection{Specular Materials}

Highly reflective objects give rise to specular reflections. A good example of this is a lake, which the sensor cannot detect. Wet pavement also does not register well with the sensor. When the Ladar scans a shiny cylindrical metallic object, only the surface of the cylinder whose normal vector is parallel with the sensor's scanning vector produces a good range reading. All other laser pulses are reflected away from the scanner. However, these reflections may bounce off of other objects and eventually return to the sensor. Consequently, ranges to reflective surfaces may appear greater than their true value. 
In nature, the primary source of specular reflections is water. Free standing water, such as lakes and puddles, almost completely reflect the laser beam from the sensor. In these cases, no return from the water's surface is detected. This appears as a "no return value" in a range images The cause of this failure is either the sensor seeing a reflection of the sky (out of range) or the water absorbing the incident beam.

The signal returned from wet surfaces varies with distance and incident angle. Wet pavement tends to disappear at close range and far away where the incident angle of the sensor beam is small. However, a good signal is returned in the mid range of $25 \mathrm{~m}$.

The EBK can measure range readings from ice and snow. However, Dornier has reported that partially thawed and refrozen snow produces a less favorable result.

\subsubsection{Vegetation}

Vegetation poses the greatest problem to obstacle detection using range data. This is because range data gives very little information about the solidity of an object or its traversability. This is especially a problem for off road driving. In addition, vegetation can obscure other vehicle hazards, such as rocks or ditches hidden in tall grass, making the hazards more difficult to detect.

\subsubsection{Fog}

The $910 \mathrm{~nm}$ wave length of the laser used by the EBK falls in the near infrared portion of the electromagnetic spectrum. It was chosen by Dornier for its good reflective properties. Being close to the visible spectrum, the laser is affected by fog. The back scattering effect caused by heavy fog causes a range value of approximately $5 \mathrm{~m}$ to be returned by the sensor. 


\section{Obstacle Detection Module}

The purpose of the obstacle detection module is to extract areas which cannot or should not be traversed by the HMMWV. Rocks, fences, trees, and steep slopes are examples of obstacle areas. Many approaches for extracting obstacles from range images have been proposed The most common approach is to fit a plane surface to a patch of points [6][8][16] in a local grid representation. For example, Hebert [6] and Kelly [10] have used the approach of fitting planar surfaces to a patch of points to detect obstacles. Their algorithms are simple and fast but are not very robust: small sensor position errors result in false indications of obstacles. We propose using differential depth and slope in sensor space in an improved, fast obstacle detector. The algorithm was inspired by Veatch and Davis [15] and Lux and Schaefer[12]. The details of this algorithm and an analysis of its robustness using actual outdoor data are given in the following subsections.

\subsection{The Obstacle Detection Algorithm}

The data acquisition time for a complete Ladar image is 0.82 seconds. The image consists of 128 vertical scan lines, each containing 64 range values. The sensor geometry was described in Section 3. Approximately half of the acquisition time is needed to read 8192 range values. The remaining time is needed for the back-scan. We process range data as they are read, rather than waiting for a full image. In this way, we can detect an obstacle as soon as the data are available.

As each scan line is read, a filter is applied to the data. The filter removes outlier points caused by specular reflections, e.g. water, or reflections within the sensor. After the data are filtered, the Cartesian $(\mathrm{x}, \mathrm{y}, \mathrm{z})$ location of each pixel in the scan line is computed in a coordinate system centered in the EBK sensor. Each pixel contains $\mathrm{x}, \mathrm{y}, \mathrm{z}$ and range(r) values which are used to compute depth derivatives and surface slopes. The criteria used for detecting obstacles are: 
1. A discontinuity in elevation exceeding some value $T$

2. The surface slope exceeding some value, $\alpha^{2}$.

These conditions are expressed mathematically in the following way:

Let $\mathrm{p}(\mathrm{i}, \mathrm{j})$, be the $\mathrm{i}^{\text {th }}$ position pixel in the $\mathrm{j}^{\text {th }}$ scan line of the EBK image.

Let $\mathrm{x}(\mathrm{i}), \mathrm{y}(\mathrm{i}), \mathrm{z}(\mathrm{i})$ be the Cartesian coordinates of this pixel.

Let $r(i)$ be the range value at this pixel.

For every $\mathrm{p}((\mathrm{k}, \mathrm{j}), \mathrm{x}(\mathrm{k}), \mathrm{y}(\mathrm{k}), \mathrm{z}(\mathrm{k})$, and $\mathrm{r}(\mathrm{k}))$ where $(\mathrm{i}-\mathrm{n})<=\mathrm{k}<=(\mathrm{i}+\mathrm{n})$

$$
\text { ( } \mathrm{n}=2 \text { in our experiments) }
$$

$\mathrm{p}((\mathrm{k}, \mathrm{j}), \mathrm{x}(\mathrm{k}), \mathrm{y}(\mathrm{k}), \mathrm{z}(\mathrm{k}), \mathrm{r}(\mathrm{k}))$ is voted an obstacle if:

$$
\begin{gathered}
|z\langle i\rangle-z\langle k\rangle| \geq T \quad \text { and } \\
\left(\langle z\langle i\rangle-z\langle k\rangle\rangle^{2} /\left[\langle x\langle i\rangle-x\langle k\rangle\rangle^{2}+\langle y\langle i\rangle-y\langle k\rangle\rangle^{2}+\langle z\langle i\rangle-z\langle k\rangle\rangle^{2}\right]\right) \geq \alpha^{2}
\end{gathered}
$$

To avoid frequent false positive detections, a local neighborhood of each pixel votes on whether or not a pixel is an obstacle. In practice, a neighborhood of five pixels is used. Each pixel in the scan line can receive up to $2 \mathrm{n}$ votes where $\mathrm{n}$ is the neighborhood size. The higher a pixel's vote, the higher the confidence that the pixel is an obstacle. If the confidence is greater than some threshold value, the pixel is labelled as an obstacle. We empirically find a confidence threshold value of 2 for our experiments.

\section{Analysis and Evaluation of the Obstacle Detection Algorithm}

Two types of tests were performed to analyze and evaluate the obstacle detection algorithm. Both scenarios involved collecting sets of Ladar data which were analyzed at a later time. The first set of tests was performed at the NIST Nike Test Site in Gaithersburg, Maryland. The obstacles 
tested were artificial, i.e., known size blocks of wood arranged in different groupings. The second set of tests was performed at the U. S. Army Aberdeen Proving Grounds (APG) in Aberdeen, Maryland. This set of data was collected at a surveyed site that included rocks and ditches.

\subsection{Artificial Obstacles}

Our objective at the Nike site was to evaluate the algorithm's ability to detect obstacles of known sizes and to determine the maximum range at which different size obstacles could be detected. The obstacles consisted of sections of $61 \mathrm{~cm} \mathrm{x} 15 \mathrm{~cm}$ lumber pre-cut into 10 $\mathrm{cm}, 15 \mathrm{~cm}$ and $20 \mathrm{~cm}$ sections. These blocks were grouped together to form obstacle groups of varying, but known, dimensions. The obstacles were placed on a road (Figure

2), and Ladar data were collected as the HMMWV approached them. Figures 3, 4 and 5

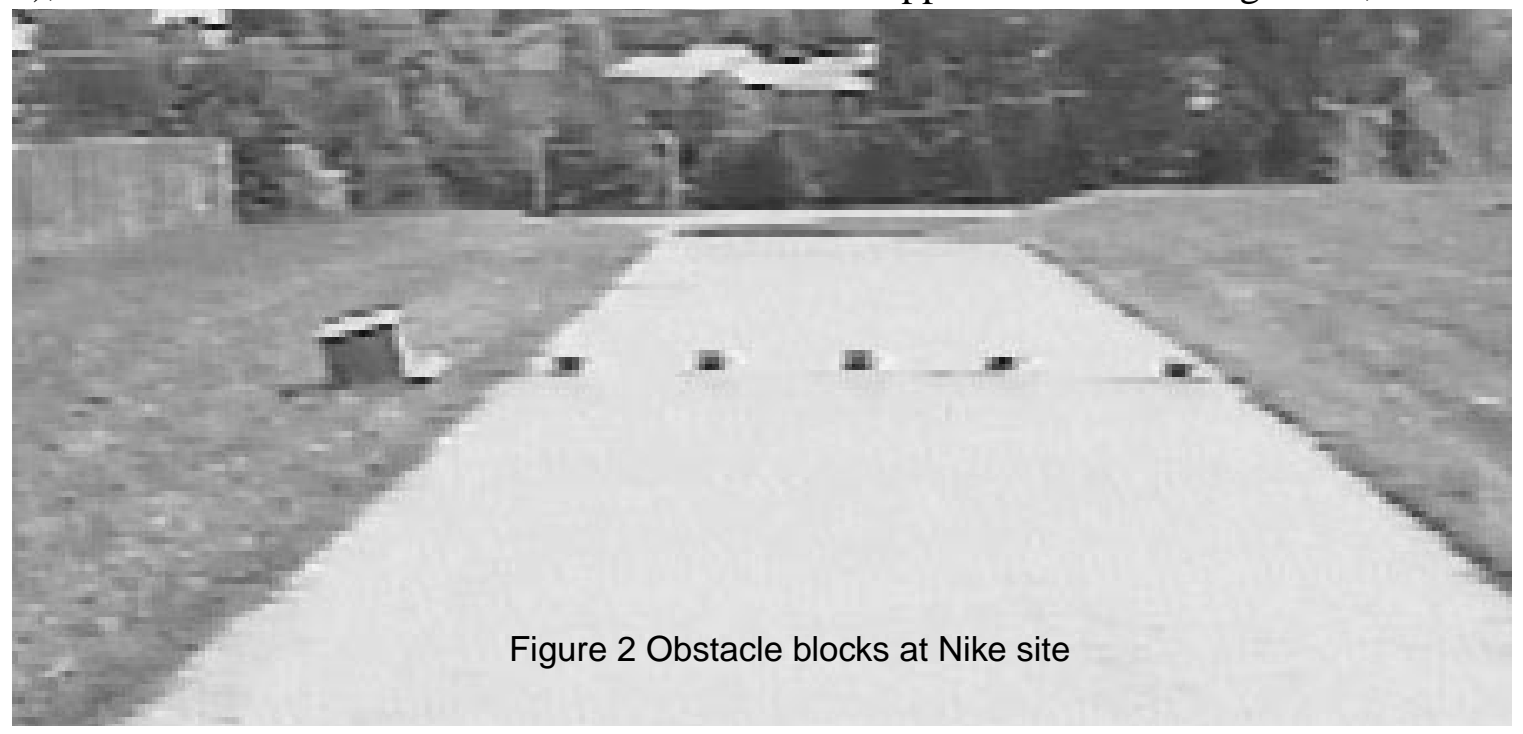

are sets of processed Ladar images showing the results of the obstacle detection algorithm on groups of different size blocks. The blocks in Figure 3 are $20 \mathrm{~cm}$ wide by 15 $\mathrm{cm}$ high. The blocks in Fig. 4 are $30 \mathrm{~cm}$ wide by $20 \mathrm{~cm}$ high. In Fig. 5, the blocks are $25 \mathrm{~cm}$ wide by $15 \mathrm{~cm}$ high. The left image in each set is the farthest range at which the 
obstacles were detected; the right image is the same set of obstacles at a closer range. In Fig. 3, only one block is detected at $17.5 \mathrm{~m}$ while all blocks are detected at $9.5 \mathrm{~m}$. In Fig. 4, 2 blocks are detected at $22 \mathrm{~m}$, and 4 blocks are detected at $13.2 \mathrm{~m}$. In Figure 5, 3 blocks are detected at $19 \mathrm{~m}$, and 4 blocks are detected at $15.7 \mathrm{~m}$.

Because of the sensor design (see Section 3), the probability of the laser beam hitting a target is a function of both the size of the target and its range from the sensor. This relationship is shown in the graphs plotted in Figure 6. In each of the plots, the horizontal axis measures range in meters from the sensor. The vertical axis is the probability that the Ladar beam will hit the target. In Figure 6a, the probability of the beam hitting the target increases to $100 \%$ at a distance of $27.5 \mathrm{~m}$ and remains at this level through the remaining valid data range. In Figure $6 \mathrm{~b}$, the probability increases to $100 \%$ at $36 \mathrm{~m}$ and remains at $100 \%$ for ranges less than $36 \mathrm{~m}$. The same is true in Figure $6 \mathrm{c}$ for ranges between $5 \mathrm{~m}$ and $47.8 \mathrm{~m}$. The target sizes in Fig. $6 \mathrm{a}, 6 \mathrm{~b}$, and $6 \mathrm{c}$ are $15 \mathrm{~cm}$ by $15 \mathrm{~cm}, 20 \mathrm{~cm} \times 20 \mathrm{~cm}$, and $25 \mathrm{~cm} \times 25 \mathrm{~cm}$ respectively. In addition to the blocks of wood, grass and trees along the side of the road are also detected as obstacle pixels. 


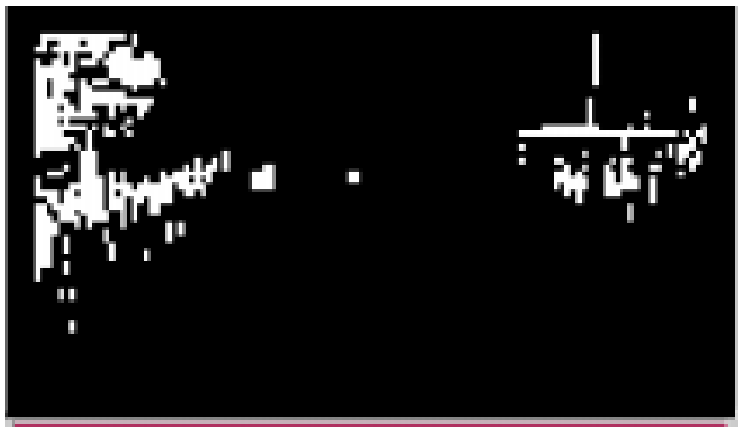

Obstacle detection at $17.5 \mathrm{~m}$

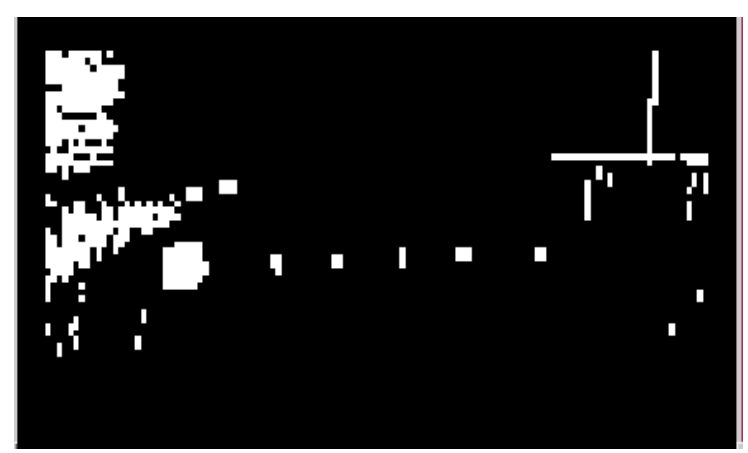

Obstacle detection at $9.5 \mathrm{~m}$

Figure 3 Target Size: $15 \mathrm{~cm} \times 20 \mathrm{~cm}$ (width $\mathrm{x}$ height)

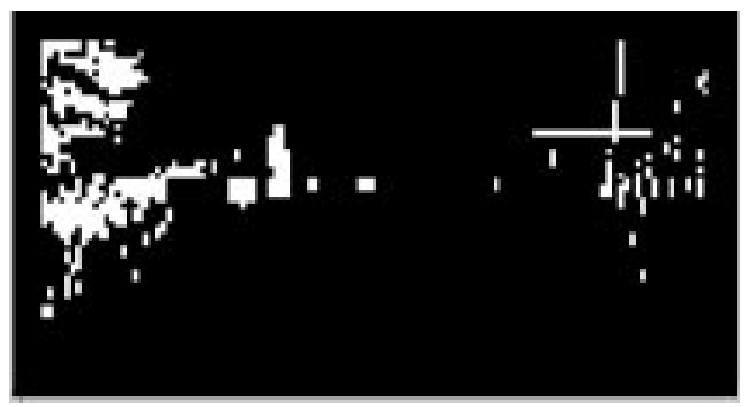

Obstacle detection at $22 \mathrm{~m}$

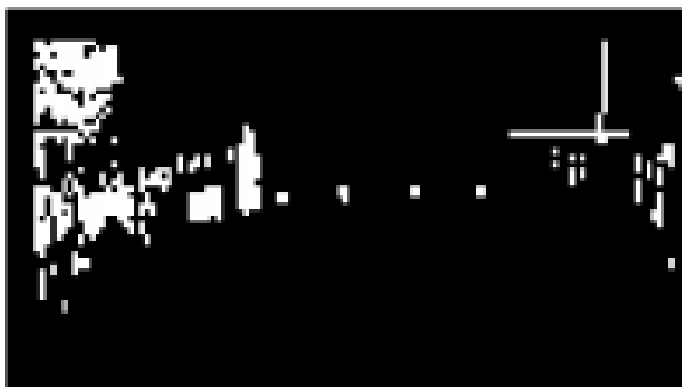

Obstacle detection at $13.2 \mathrm{~m}$

Figure 4 Target Size $30 \mathrm{~cm} \times 20 \mathrm{~cm}$

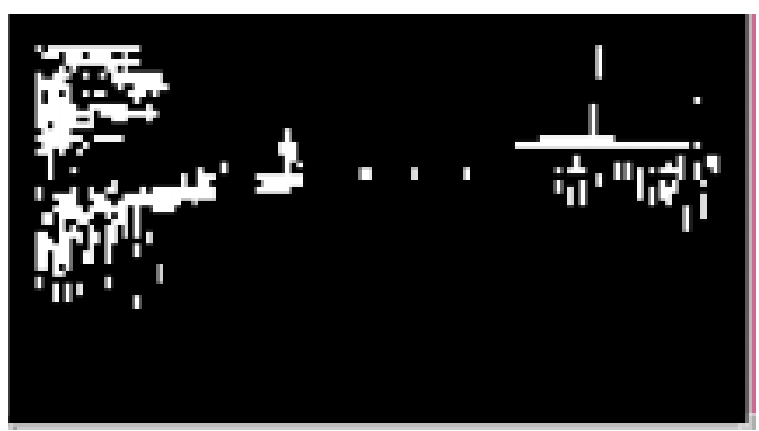

Obstacle detection at $19 \mathrm{~m}$

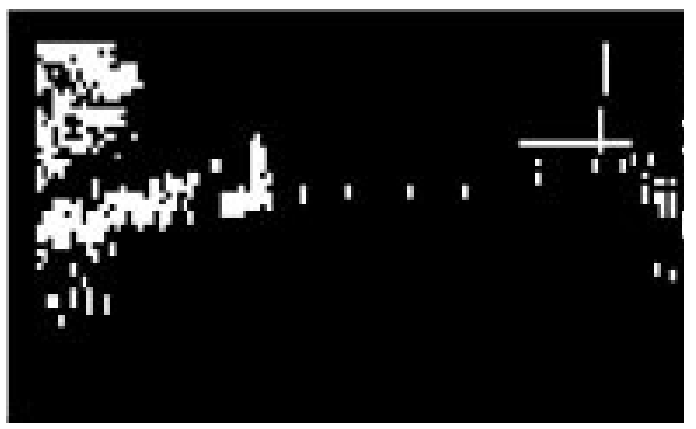

Obstacle detection at $15.7 \mathrm{~m}$

Figure 5 Target Size: $25 \mathrm{~cm} \times 15 \mathrm{~cm}$ 


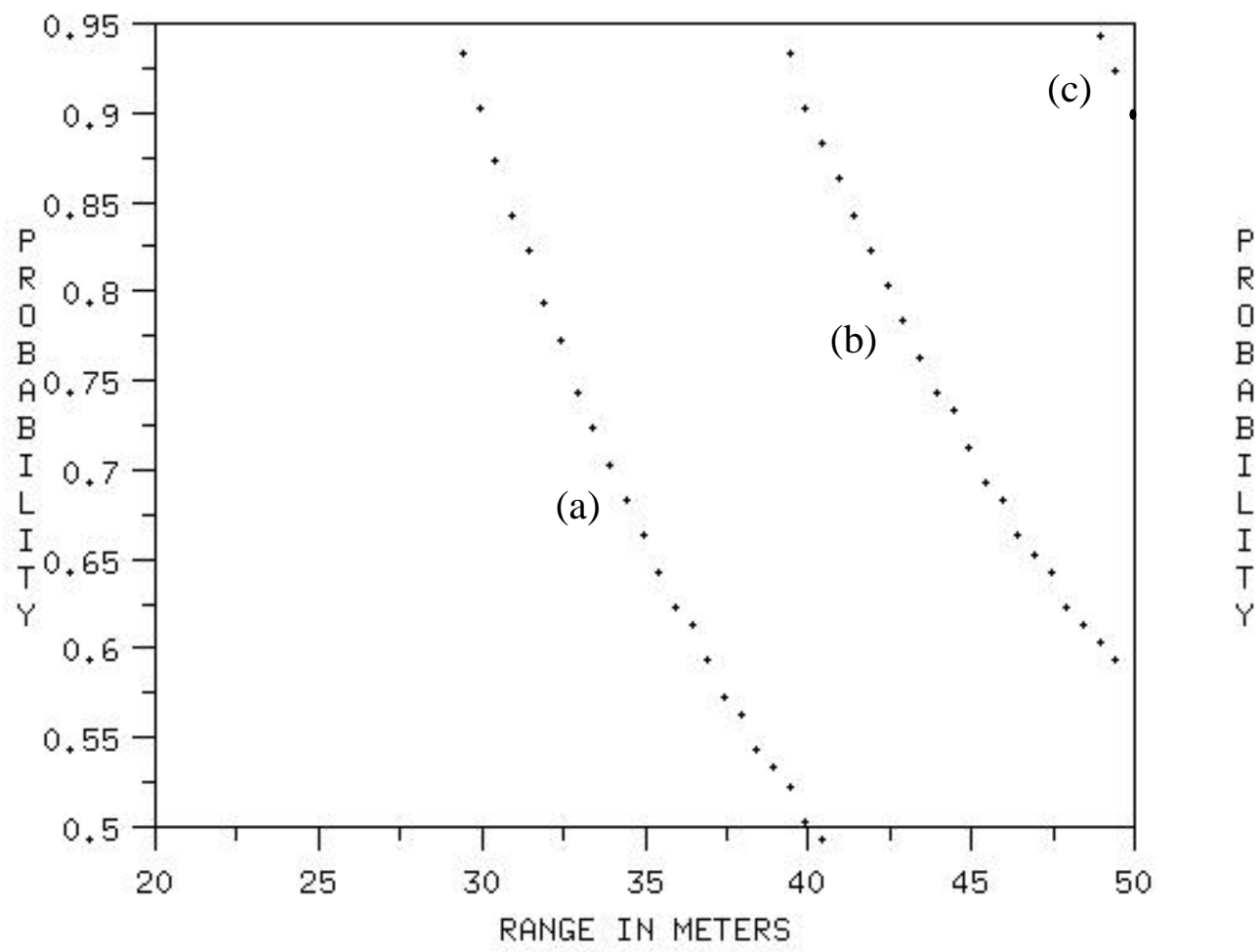

Figure 6: Probability plots of ladar beam hitting target.

(a) Target size $15 \mathrm{~cm} \times 15 \mathrm{~cm}$ (b) Target size $20 \mathrm{~cm} \times 20 \mathrm{~cm}$ (c) Target size $25 \mathrm{~cm} \times 25 \mathrm{~cm}$

\subsection{Natural Obstacles}

In this section, we describe our analysis of natural obstacles. Our objective was to determine the farthest range at which pixels representing depressions could be detected. The data collection site was the Perryman site, located in the U. S. Army Aberdeen Proving Grounds in Aberdeen, Maryland. In addition to the Ladar data, sets of images from 3 pairs of stereo cameras were also collected. ${ }^{2}$ The stereo data were analyzed by scientists at NASA's Jet Propulsion Laboratory (JPL) using algorithms designed for stereo inputs [13]. This report discusses only

2. A complete description of the data collection can be found at: http://isd.cme.nist.gov/staff/coombs/proj/mobility/apg-data/doc/datanotes.html. 
the results of the Ladar obstacle detection algorithm.

Figure 7 is a map of the sections of Perryman in which negative obstacle (ditch) data were collected. The ditches were dug by a back hoe. Ditch 1 is $0.61 \mathrm{~m}$ wide; Ditch 2 is $1.22 \mathrm{~m}$ wide, Ditch 3 is $1.83 \mathrm{~m}$ wide, and Ditch 4 is $2.44 \mathrm{~m}$ wide. The lengths of all the ditches are the same. This data was used to evaluate the effectiveness of the algorithm in detecting

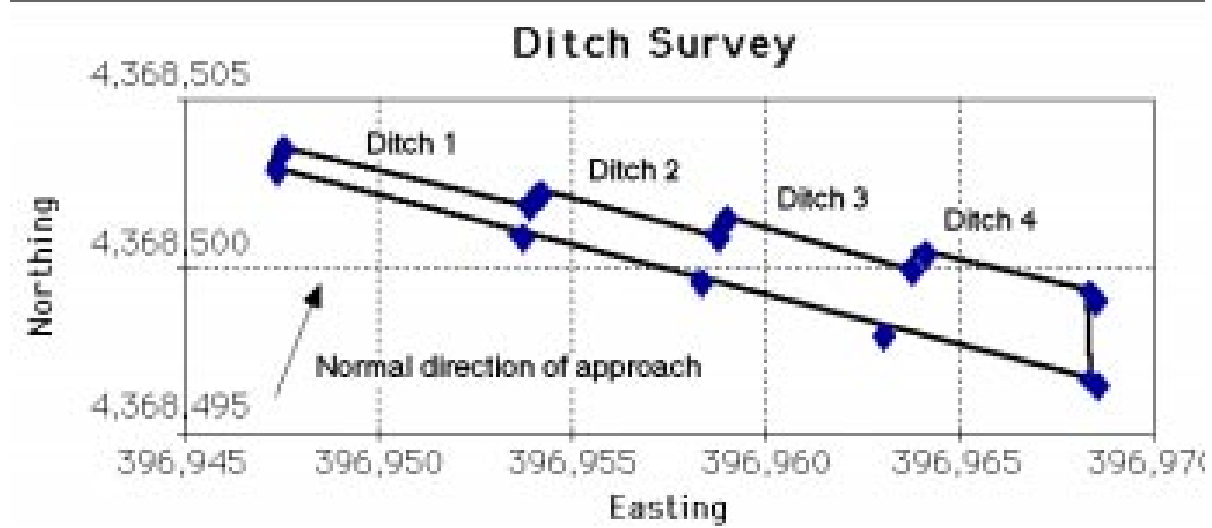

Figure 7 Ditch Survey Site

negative obstacles (depressions in the ground plane). Figure 8 is a black and white image, digitized from video tape, showing the site referred to in the ditch survey map. The viewing direction in Figure 8 is along the direction of the approach vector in Figure 7. Data were collected at different times of day under varying weather conditions. A

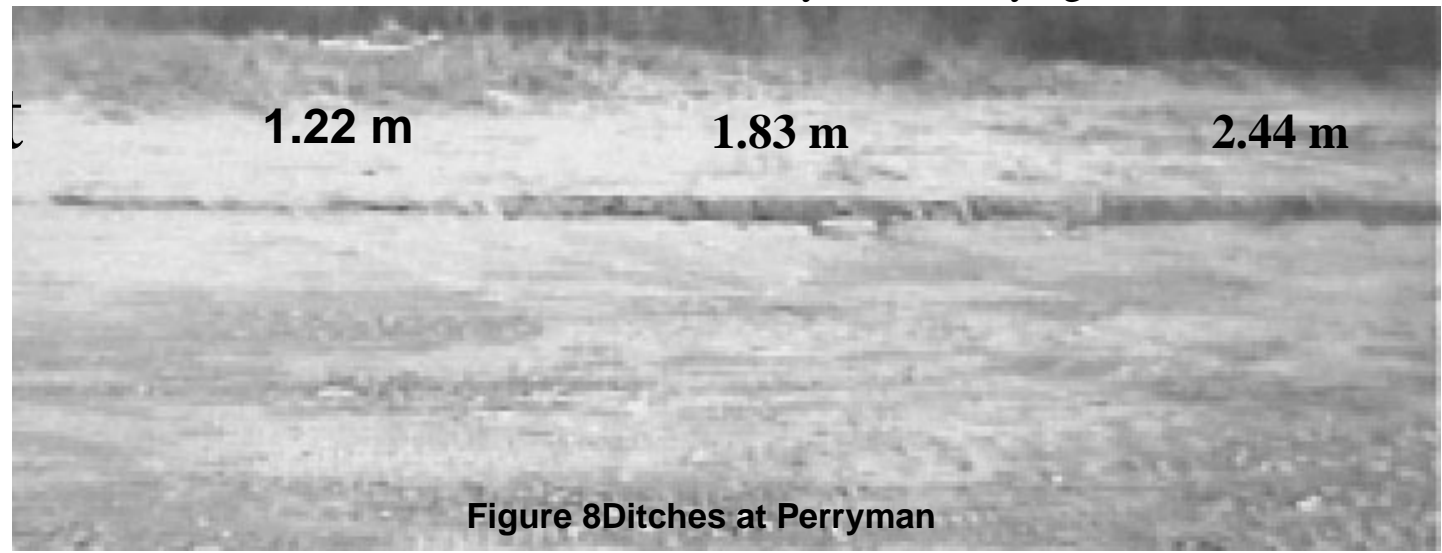

decrease in daylight did not affect the Ladar data which performed at the same level 
during dawn, daylight, and evening hours. For each of the ditches, the HMMWV was driven at speeds of 8 kilometers per hour $(\mathrm{km} / \mathrm{h}), 16 \mathrm{~km} / \mathrm{h}$, and $24 \mathrm{~km} / \mathrm{h}$ The vehicle's approach to the ditches also varied: some runs were perpendicular to the ditches; some were oblique. The output of the obstacle detection algorithm was qualitatively evaluated. Grouping of obstacle pixels into objects is planned at a future time and was not a factor in this evaluation. Figure 9 is a set of images showing the unprocessed Ladar data (Figure 9a) and the processed data (Figure 9b) of a sample image taken from the "ditch 1" data set.The raw data is encoded as grey scale values such that darker grey values represent ranges farther from the sensor. White pixels in the processed image represent pixels labelled as obstacle points. Although it is very difficult to recognize the ditch in the raw image, the ditch pixels are detected in the processed image The ditch is $10 \mathrm{~m}$ from the vehicle in Figure 9.

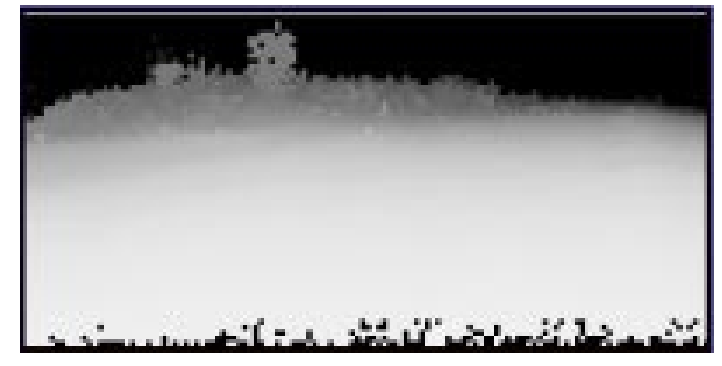

(a)

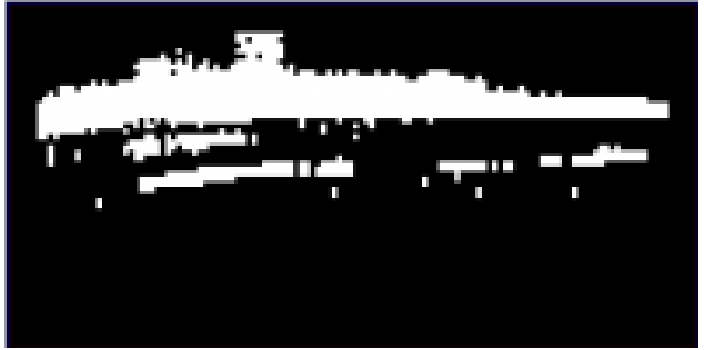

(b)

Figure 9 Ditch 1: Raw and processed ladar data

Figure 10 is another example of negative obstacle detection. This image set shows raw and processed data from the "ditch 4" data sequence. Again, the pixels associated with the ditches are extracted. In this figure, the ditch is $15 \mathrm{~m}$ from the vehicle.

Table 2 shows the results of the obstacle detection algorithm on the ditch data as the vehicle approached the ditches in the normal direction. The distances, measured in meters, represent the farthest distance at which the algorithm extracted pixels representing 


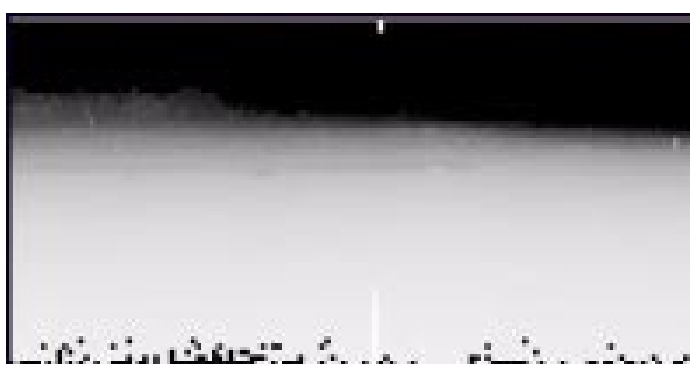

(a)

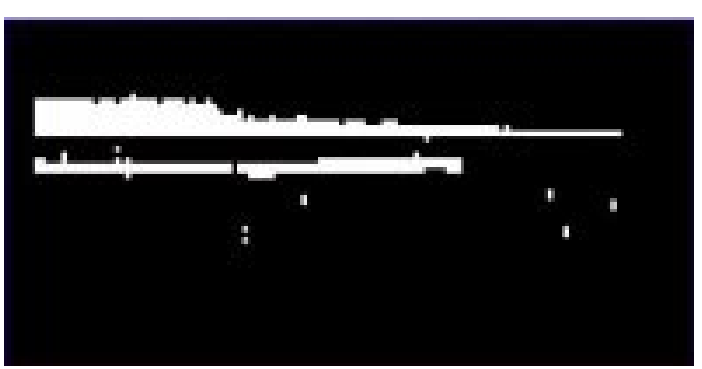

(b)

Figure 10 Ditch 4: Raw and processed ladar data

ditch data. The pixels associated with the ditch are qualitatively recognizable to a trained eye.

As an aid to understanding these values, it is helpful to understand that the minimum stopping distance required for a vehicle travelling at 5 m.p.h. (assuming a relatively flat ground surface) is approximately 10 meters. At 10 m.p.h., the stopping distance is 20 meters, and at 15 m.p.h., it is 30 meters.

Table 2 NEGATIVE OBSTACLE DETECTION- NORMAL APPROACH

\begin{tabular}{|r|c|c|c|c|}
\hline & Ditch 1 & Ditch 2 & Ditch 3 & Ditch 4 \\
\hline \hline $5 \mathrm{mph}$. & $10.0 \mathrm{~m}$ & $19.0 \mathrm{~m}$ & $17.0 \mathrm{~m}$ & $23.0 \mathrm{~m}$ \\
\hline $10 \mathrm{mph}$. & $10.0 \mathrm{~m}$ & $14.0 \mathrm{~m}$ & $20.0 \mathrm{~m}$ & $20.7 \mathrm{~m}$ \\
\hline $15 \mathrm{mph}$. & $9.5 \mathrm{~m}$ & $15.0 \mathrm{~m}$ & $20.5 \mathrm{~m}$ & $21.2 \mathrm{~m}$ \\
\hline
\end{tabular}

Figure 11 is a map of the area of Perryman containing the rock obstacles. The rocks were placed both on-road and off-road. The rocks labelled "rock 1" and "rock 2" are approximately $15 \mathrm{~cm}$ high and $25 \mathrm{~cm}$ wide. Rocks 3 and 4 are approximately $30 \mathrm{~cm}$ high and $30 \mathrm{~cm}$ wide. Rock 5 is approximately $45 \mathrm{~cm}$ high and $30 \mathrm{~cm}$ wide. The rock cluster is 
approximately $45 \mathrm{~cm}$ high and $100 \mathrm{~cm}$ wide.

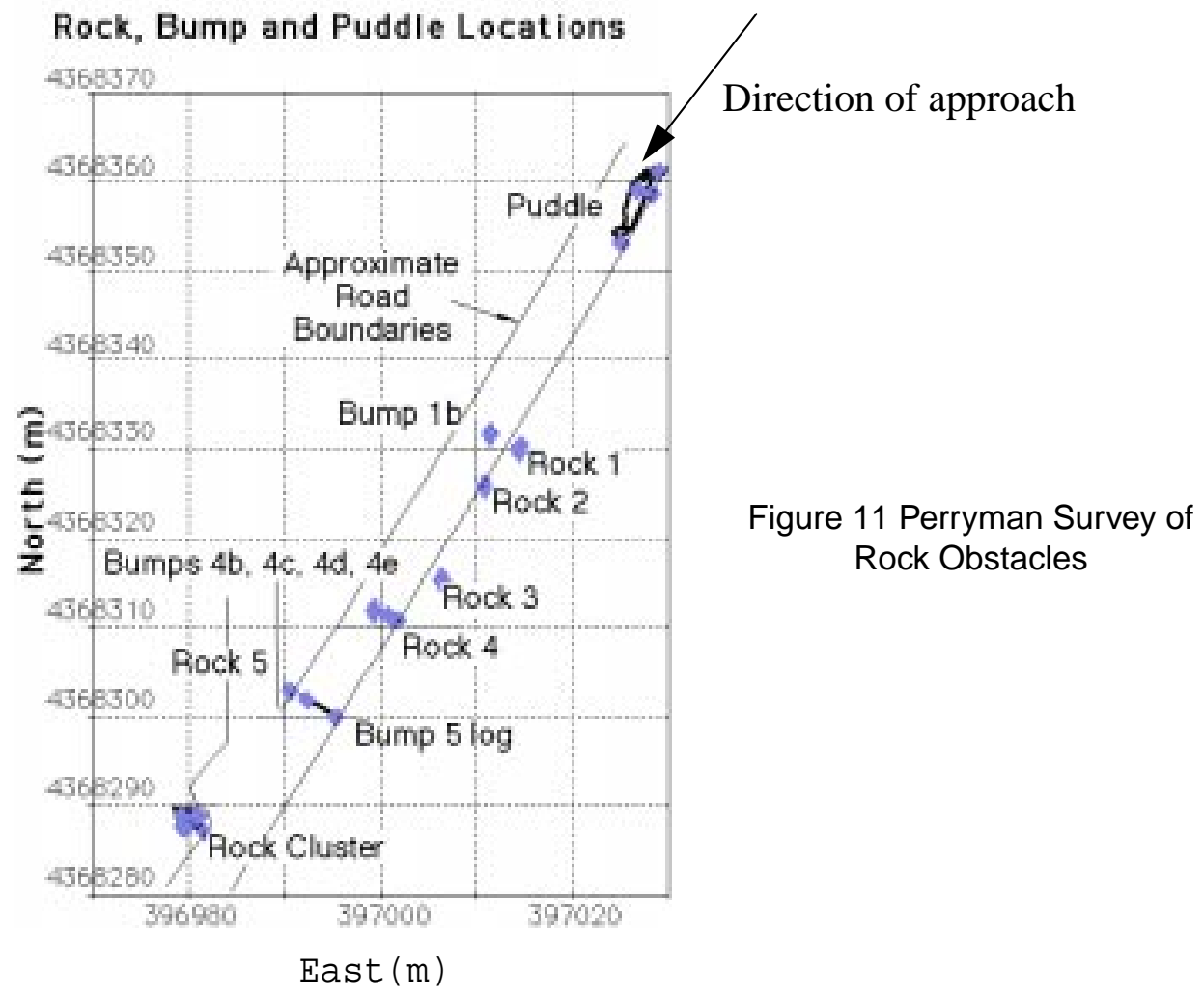


Figure 12 is a video image showing rocks $1,2,3$ and 4.

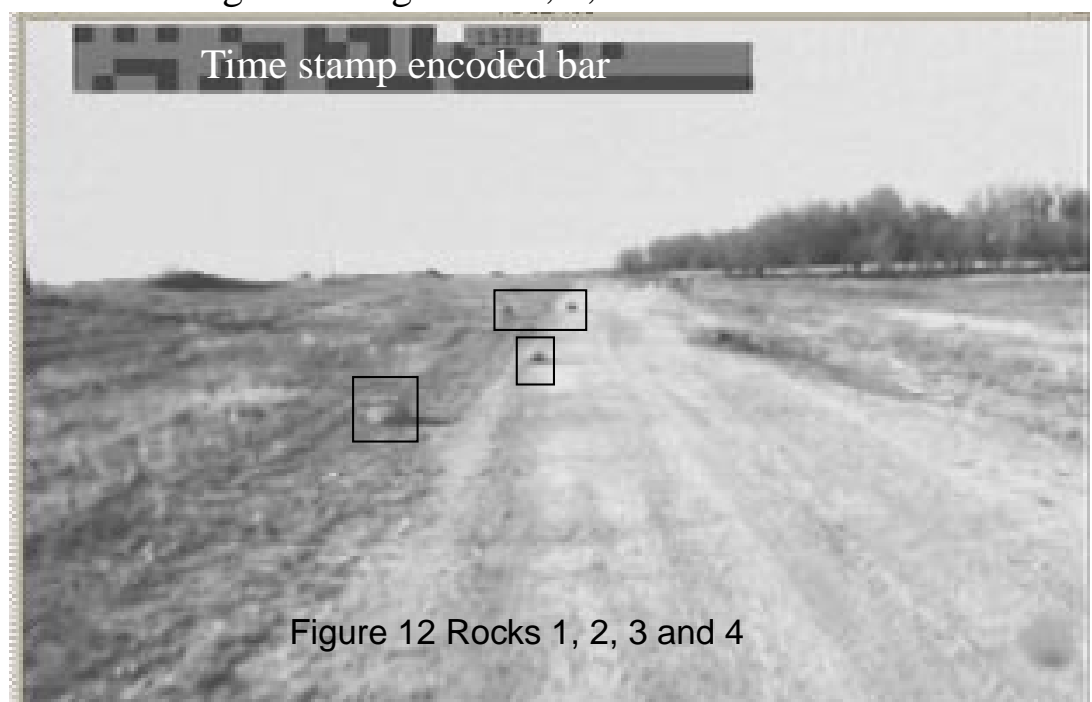

Figure 13 is a magnified video image showing the rock cluster.

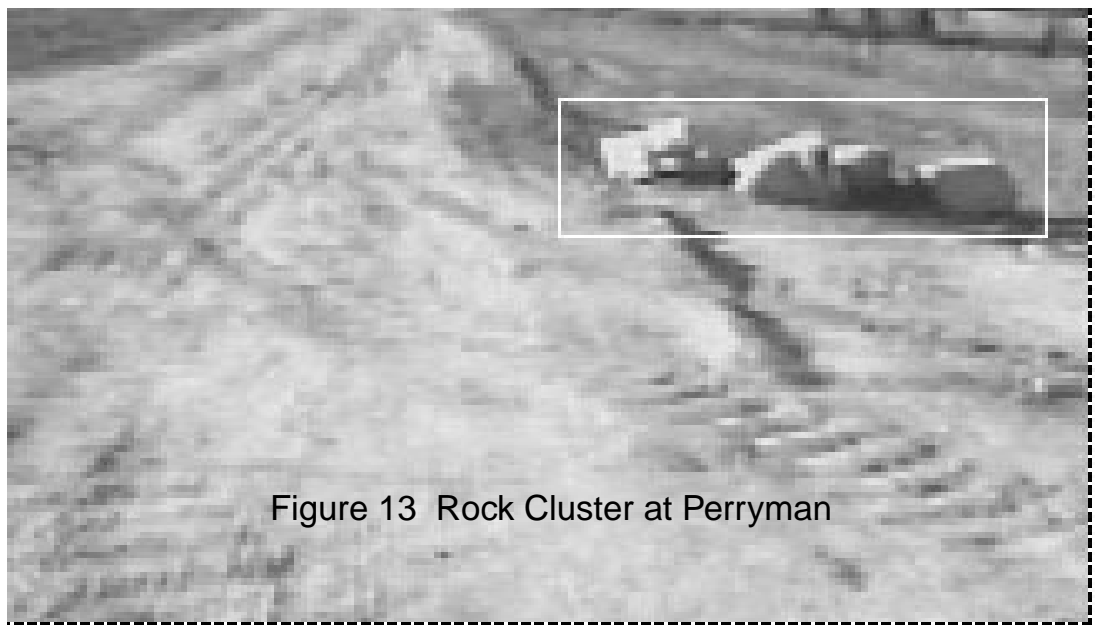

Figure 14a shows a Ladar scene of rocks 1 and 2 and Figure 14b the obstacles detected in this image. Rocks 1 and 2 are highlighted by white boxes in the right image.

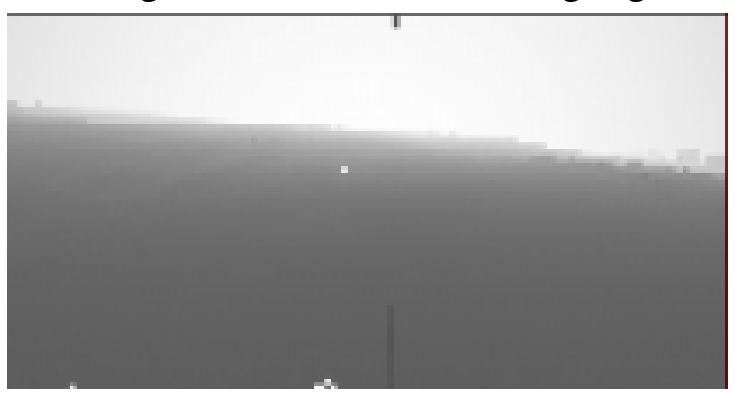

(a)Raw ladar data

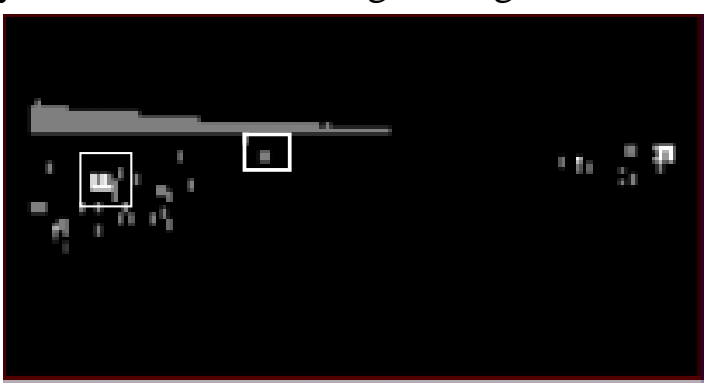

(b) Rock 1 at a distance of $11.5 \mathrm{~m}$ Rock 2 at a distance of $19.2 \mathrm{~m}$

Figure 14 (a) Raw ladar image of rocks 1 and 2 
Figure 15 shows raw (Fig. 15a) and processed (Fig. 15b) Ladar image data for rocks 3 and 4 with boxes highlighting the rocks.

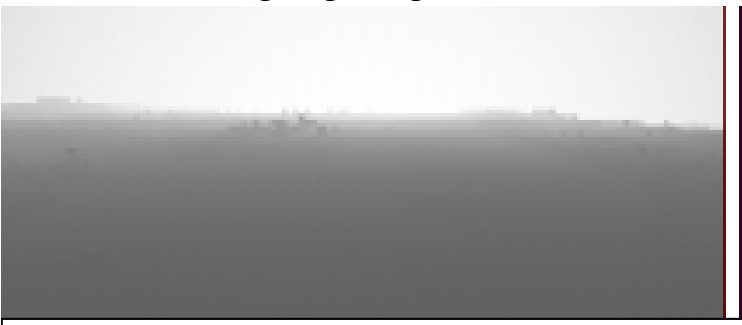

(a)Raw ladar data

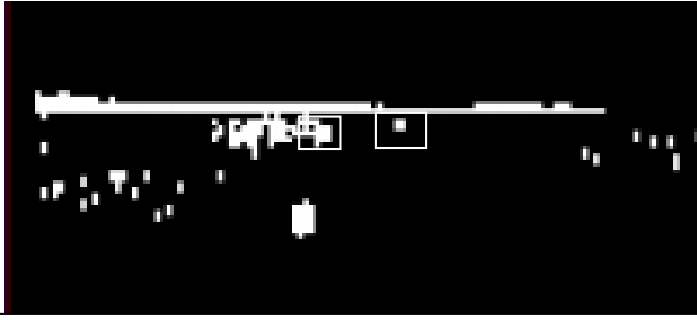

(b) Rock 3 at a distance of $18.4 \mathrm{~m}$ Rock 4 at a distance of $25.0 \mathrm{~m}$

Figure 15 Rocks 3 and 4

Figure $16 \mathrm{a}$ is a Ladar scene of the rock cluster. Figure $16 \mathrm{~b}$ shows the extracted obstacle points. The direction of approach for Figs. 14, 15, and 16 is from Northeast to Southwest in Fig. 11. Table 3 is a summary of the results of applying the obstacle detection

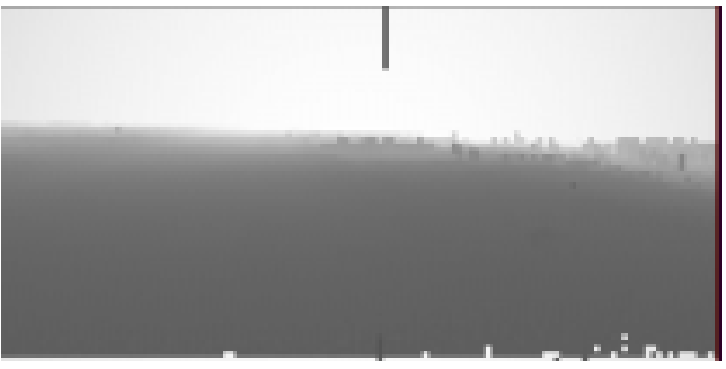

(a) Raw ladar data

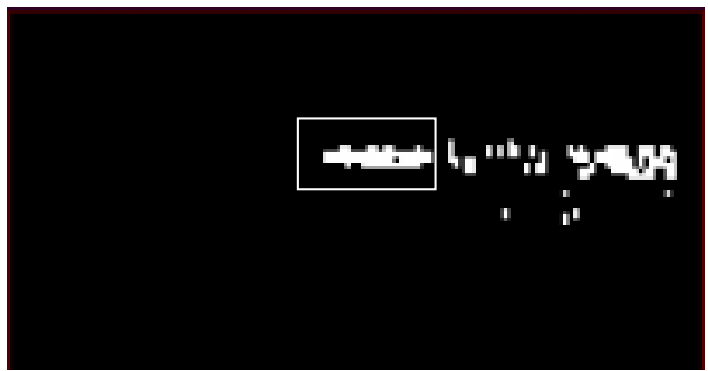

(b) Rock cluster at a distance of $25 \mathrm{~m}$

Figure 16 Rock Cluster

algorithm to the data sets collected at different times of day. The variability of detection is a result of the following factors:

(1) Data acquisition rate (1 image per second).

(2) Relationship between vehicle velocity and the time an image is acquired. If the vehicle is moving quickly, the scanning Ladar beam may miss the obstacle completely.

(3) Difficulty in identifying the targeted obstacle when it is hidden in tall grass. 
Table 3 Detection of Positive Obstacles

\begin{tabular}{|c|c|c|c|c|c|c|}
\hline & Rock1 & Rock2 & Rock3 & Rock4 & Rock5 & $\begin{array}{c}\text { Rock } \\
\text { Clump }\end{array}$ \\
\hline \hline $5 \mathrm{mph}$ & $13.9 \mathrm{~m}$ & $20.2 \mathrm{~m}$ & $18.6 \mathrm{~m}$ & $25.0 \mathrm{~m}$ & $12.8 \mathrm{~m}$ & $31.8 \mathrm{~m}$ \\
\hline $10 \mathrm{mph}$ & $13.2 \mathrm{~m}$ & $20.5 \mathrm{~m}$ & $22.3 \mathrm{~m}$ & $24.1 \mathrm{~m}$ & $17.0 \mathrm{~m}$ & $26.0 \mathrm{~m}$ \\
\hline $15 \mathrm{mph}$ & $15.0 \mathrm{~m}$ & $23.2 \mathrm{~m}$ & $22.2 \mathrm{~m}$ & $26.6 \mathrm{~m}$ & $13.7 \mathrm{~m}$ & $30.6 \mathrm{~m}$ \\
\hline
\end{tabular}

The need for recognizing and classifying obstacles is clear from the outputs shown in these figures. In addition to the rocks and/or rock cluster, the algorithm has flagged tall grass, bushes, and background trees as obstacles. Our future work includes plans to fuse Ladar images with video images to generate windows of interest in the video images. Classification of obstacles will be based on the information in the video windows.

\section{Obstacle Map and List Module}

We have adapted Kelly's [10] grid obstacle map for representing obstacles for path planning and vehicle control. The map representation contains terrain surface slopes, uncertainty measures, average depth information, and a list of obstacles for every grid in the map. The path planning algorithm currently implemented in the 4D-RCS system [11] uses only a subset of the information contained in the map. It generates traversability paths from the map by using a binary flag indicating whether or not the grid is an obstacle, a timestamp indicating when an obstacle was last updated, and an obstacle confidence value.

\section{Conclusion}

We have described an algorithm designed to detect obstacles in Ladar imagery. The 
algorithm is one of a suite of algorithms being tested in order to evaluate different sensors that might be used for obstacle detection in the UGV program. We have characterized the Ladar sensor being used, and described its performance on different types of surfaces.

In order to evaluate the obstacle detection algorithm, we collected extensive sets of Ladar data. These data were used as input for the obstacle detection algorithm. The algorithm was able to detect both positive (rocks) and negative (ditches) obstacles. Detectability was determined to be a function of both the range to the obstacle and the size of the obstacle. The results of the evaluation tests are reported.

The obstacle detection algorithm was also tested in a real-time demonstration as part of the 4D-RCS control system. In conjunction with the planner module and behavior generation modules [1], we were able to detect obstacles on both the NIST grounds and the NIKE test site while driving.

\section{Acknowledgments}

This work was sponsored by the Joint Robotics Program of the Under Secretary of Defense for Acquisition and Technology (JRP OUSD(A\&T)) and monitored by the U. S. Army Research Laboratory. We thank the members of the Intelligent Autonomous Vehicles Group at NIST, ARL, the Toole Army Depot, the Aberdeen Test Center, and JPL for their efforts in the Perryman data collection. In particular, we thank Maris Juberts, Martin Herman, David Coombs, Billibon Yoshimi, Karl Murphy, Jim Gilsinn, Larry Matthies, Todd Litman, Ken Owens, Tim Vong, Chuck Shoemaker, Jon Borenstein, Gary Haas, Fred Eldridge, Ish Sherali, and Bob Brooks. We apologize to anyone inadvertently left off this list. 


\section{References}

[1] Albus, James, “4-D/RCS Version 1.0, A Reference Model Architecture for Demo III,” TBP

[2] S. Baten, R. Mandelbaum, M. Lutzeler, P. Burt, E. D. Dickmanns, "Area-based stereo image processing within the 4D architecture for autonomous off-road navigation," AutoNav Meeting, FL, January, 1998.

[3] Dornier GmbH, "Evaluation Package User's Guide."

[4] Dornier GmbH, "Transformation of EBK range Images to 3-D Images in a Sensor Coordinate System."

[5] Hebert, M. and Kanade, T. , "First Results on Outdoor Scene Analysis Using Range Data," Proceedings: Image Understanding Workshop (Miami Beach, FL, December 9--10, 1985), pp. 224-231, Morgan Kaufmann, 1985.

[6] Hebert, M. and Ponce, J., "A New Method for Segmenting 3-D Scenes into Primitives," 6th International Conference on Pattern Recognition, pp836-838, Oct. 1982.

[7] M. Hebert, C. Thorpe, and A. Sentz, Intelligent Unmanned Ground Vehicles, Autonomous Navigation Research at Carnegie Mellon, Kluwer Academic Researchers, 1997.

[8] Hoffman, R. and Jain, A.K., "Segmentation and Classification of Range Images," PAMI(9), No. 5, pp. 608-620., Sept. 1987.

[9] Kalman, R.E., "A new approach to linear filtering and prediction problems," Trans. ASME, Series D, J. Basic Eng., V. 82, pp. 35 - 45, 1960.

[10] Kelly, A., "An Intelligent, Predictive Control Approach to the High-Speed Cross-Country Autonomous Navigation Problem," Carnegie Mellon University, 1995

[11] Lacaze, A., Albus, J., Meystel, A., "Planning in the Hierarchy of NIST-RCS for Manufacturing," Proceedings of the International Conference on Intelligent Systems: A Semiotic Perspective, Gaithersburg, MD, October 20-23, 1996.

[12] Lux, P.W. and Schaefer, C.H., "Range Imaging for Autonomous Navigation of Robotic Land Vehicles,” Dornier Lufthahrt, Signal Processing 22, pp299-311, 1991.

[13] Matthies, L., Litwin T., Owens, K. Rankin, A., et. al., "Performance Evaluation of UGV Obstacle Detection with LADAR and CCD/FLIR Stereo Vision," Proceedings of SPIE AeroSense Conference, April, 1998.

[14] Murphy, K., Legowik, S., "GPS Aided Retrotraverse For Unmanned Ground Vehicles," Proceedings of the SPIE 10th Annual AeroSense Symposium, Conference 2738, Navigation and Control, Technologies for Unmanned Systems, Orlando, FL, April 1996.

[15] Veatch, P. A. and Davis, L. S., "Efficient Agorithms for Obstacle Detection Using Range data," Computer Vision, Graphics, and Image Processing, 50(1), pp. 50-74, April 1990.

[16] Yang, H.S. and Boyer, K.L. and Kak, A.C., "Range Data Extraction and Interpretation by Structural Light," pp 199-205, Conference on Artificial Intelligence Applications, 1984. 